\title{
Schur Convexity Condition for Novel Ratio of Difference of Means
}

\author{
Sreenivasa Reddy Perla \\ The Oxford College of Engg \\ Dept of Mathematics, VTU \\ Bangalore, Karnataka. India. \\ srireddy_sri@yahoo.co.in

\section{Padmanabhan S} \\ RNSIT \\ Dept of Mathematics, VTU \\ Bangalore, Karnataka. India. \\ padmanabhanrnsit@gmail.com
}

\author{
K M Nagaraja \\ JSSATE \\ Dept of Mathematics, VTU \\ Bangalore, Karnataka. India. \\ nagkmn@gmail.com \\ Padmanava Samanta \\ Dept of Mathematics \\ Berhampur University, \\ Odissa.India. \\ dr.pns.math@gmail.com
}

\begin{abstract}
In this paper, we study the different kind of convexities like Schur, Schur Geometric and Schur Harmonic convexities for novel ratio of difference of means.
\end{abstract}

Keywords: Schur convexity, Schur harmonic convexity, Schur Geometric convexity, ratio of difference of means and inequality.

\section{INTRODUCTION}

The well-known means in literature such as arithmetic mean, geometric mean harmonic mean and contra harmonic mean are presented by pappus of Alexandria. In Pythagorean School on the basis of proportion and also some of the other means like Heron mean and Centriodal Mean are defined as follows [1,2] and some interesting results on above said means are discussed in [3-8].

For two positive real numbers $a \& b$;

$$
\begin{aligned}
& \text { Arithmetic Mean }=A(a, b)=\frac{a+b}{2} \quad \text { Geometric Mean }=G(a, b)=\sqrt{a b} \\
& \text { Harmonic Mean }=H(a, b)=\frac{2 a b}{a+b} \quad \text { Contra Harmonic Mean }=C(a, b)=\frac{a^{2}+b^{2}}{a+b} \\
& \text { Heron mean }=H_{e}(a, b)=\frac{a+\sqrt{a b}+b}{3} \text { and Centriodal Mean } C_{d}(a, b)=\frac{2\left(a^{2}+a b+b^{2}\right)}{3(a+b)}
\end{aligned}
$$

Jamal Rooin and Mehdi Hassni [9], introduced the homogeneous functions $f(x)$ and $g(x)$, also established some convexity results and refinements to Ky-Fan-type inequalities.where

$$
f(x)=\frac{a^{x}-b^{x}}{c^{x}-d^{x}} \text { and } g(x)=\ln \frac{a^{x}-b^{x}}{c^{x}-d^{x}} \text { for } x \in(-\infty, \infty) \text { and } a>b \geq c>d .
$$

In [10], authors studied the convexity(concavity) of the following ratio of difference of means.

$$
M_{C A G H}(a, b)=\frac{C(a, b)-A(a, b)}{G(a, b)-H(a, b)} \quad M_{A H_{e} G H}(a, b)=\frac{A(a, b)-H_{e}(a, b)}{G(a, b)-H(a, b)}
$$




$$
\begin{array}{lll}
M_{C H_{e} G H}(a, b)=\frac{C(a, b)-H_{e}(a, b)}{G(a, b)-H(a, b)} & M_{C_{d} A G H}(a, b)=\frac{C_{d}(a, b)-A(a, b)}{G(a, b)-H(a, b)} \\
M_{C_{C A H_{e} G}(a, b)=\frac{C(a, b)-A(a, b)}{H_{e}(a, b)-G(a, b)}} & \text { and } & M_{C C_{d} G H}(a, b)=\frac{C(a, b)-C_{d}(a, b)}{G(a, b)-H(a, b)}
\end{array}
$$

Some fruitful results related to Schur convexities were also found in [11-22].

In this paper, we study the Schur, Schur harmonic and Schur geometric convexity of ratio of means $M_{C A G H}(a, b), M_{A H_{e} G H}(a, b), M_{C H_{e} G H}(a, b) \& M_{C A H_{e} G}(a, b)$ and some applications of these ratio of difference of means.

\section{Preliminary Results and Definitions}

In 1923, the Schur Convex function was introduced by I Schur, and proved many important applications to analytic inequalities. In 2003, X. M. Zhang propose the concept of Schurgeometrically convex function which is an extension of Schur-convexity function. In recent years, the Schur convexity, Schur geometrically convexity and Schur harmonic convexity have attracted the attention of a considerable number of mathematicians ([11],- [21]). For convenience of readers, we recall some definitions as follows:

Definition 2.1.[3,7] Let $x=\left(x_{1}, x_{2}, x_{3}, \ldots \ldots . x_{n}\right)$ and $y=\left(y_{1}, y_{2}, y_{3}, \ldots \ldots . y_{n}\right) \in R^{n}$

1. Let $x$ is said to be majorized by $y$ (in symbol $x<y$ ) $\sum_{i=1}^{k} x_{i} \leq \sum_{i=1}^{k} y_{i} \quad$ for $k=1,2,3 \ldots n$ and $\sum_{i=1}^{k} x_{i}=\sum_{i=1}^{k} y_{i} \quad$ where $x_{1} \geq \ldots \ldots . . \geq x_{n}$ and $y_{1} \geq \ldots \ldots . \geq y_{n}$ are rearrangement of $x$ and $y$ in descending order.

2. $\Omega \subseteq R^{n}$ The function $\varphi: \Omega \rightarrow R^{n}$ is said to be schur convex function on $\Omega$ if $x<y$ on $\Omega$ implies $\varphi(x) \leq \varphi(y) . \varphi$ is said to be a Schur concave function on $\Omega$ if and only if $-\varphi$ is Schur convex.

Definition 2.2.[22] Let $x=\left(x_{1}, x_{2}, x_{3}, \ldots \ldots . x_{n}\right)$ and $y=\left(y_{1}, y_{2}, y_{3}, \ldots \ldots . y_{n}\right) \in R^{n}+\Omega \subseteq R^{n}$ is called geometrically convex set if $x_{1}{ }^{\alpha} y_{1}{ }^{\beta} \ldots \ldots x_{n}{ }^{\alpha} y_{n}{ }^{\beta} \in R^{n}$ for all $x$ and $y$ where $\alpha, \beta \in[0,1]$ with $\alpha+\beta=1$. Let $\Omega \subseteq R^{n}{ }_{+}$The function $\varphi: \Omega \rightarrow R_{+}{ }^{n}$ is said to be schur geometrically convex function on $\Omega$ if $\left(\ln x \ldots \ldots . \ln x_{n}\right)<\left(\begin{array}{lll}\ln y & \ldots \ldots . . \ln y_{n}\end{array}\right)$ on $\Omega$ implies $\varphi(x) \leq \varphi(y)$. Let $\varphi$ is said to be a Schur geometrically concave function on $\varphi$ if and only if $-\varphi$ is Schur geometrically convex.

Definition 2.3.[3,7] The set $\Omega \subseteq R^{n}$ is called symmetric set if $x \in \Omega$ implies $p x \in \Omega$ for every $n \times n$ permutation matrix $p$. The function $\varphi: \Omega \rightarrow R^{n}$ is said to be symmetric if every permutation matrix $p ; \varphi(p x)=\varphi(x)$. for all $x \in \Omega$.

we introduce the ratio of difference of means as follows:

$$
\frac{M_{C A G H}}{M_{A H_{e} G H}}(a, b)=\frac{C(a, b)-A(a, b)}{A(a, b)-H_{e}(a, b)} \quad \frac{M_{C C_{d} G H}}{M_{C_{d} A G H}}(a, b)=\frac{C(a, b)-C_{d}(a, b)}{C_{d}(a, b)-A(a, b)}
$$




$$
\begin{array}{ll}
\frac{M_{C_{d} A G H}}{M_{A H_{e} G H}}(a, b)=\frac{C_{d}(a, b)-A(a, b)}{A(a, b)-H_{e}(a, b)} & \frac{M_{C C_{d} G H}}{M_{A H_{e} G H}}(a, b)=\frac{C(a, b)-C_{d}(a, b)}{A(a, b)-H_{e}(a, b)} \\
\frac{M_{C_{d} A G H}}{M_{A H_{e} G H}}(a, b)=\frac{C_{d}(a, b)-A(a, b)}{A(a, b)-H_{e}(a, b)} & \frac{M_{C C_{d} G H}}{M_{A H_{e} G H}}(a, b)=\frac{C(a, b)-C_{d}(a, b)}{A(a, b)-H_{e}(a, b)}
\end{array}
$$

Lemma 2.1.[3,7] Let $\Omega \subseteq R^{n} \varphi: \Omega \rightarrow R$ is symmetric and convex function. Then $\varphi$ is Schur convex on $\Omega$.

Lemma 2.2.[9] For $a>b \geq c>d$. the function $f(x)=\frac{a^{x}-b^{x}}{c^{x}-d^{x}}$ where $x \in(-\infty, \infty)$ is

i) Convex, if $a d-b c>0$

ii) Concave if $a d-b c<0$

iii) Equality holds if $a d-b c=0$.

Lemma 2.3.[22] Let $\Omega \subseteq R^{n}$ be symmetric with non empty interior convex set and let $\varphi: \Omega \rightarrow R_{+}$be continuous on $\Omega$ and differentiable on $\Omega^{0}$ If $\varphi$ is symmetric on $\Omega$ and for any on $x=\left(x_{1}, x_{2}, x_{3}, \ldots \ldots . . . x_{n}\right) \in \Omega^{0}$. Then $\varphi$ is

(i) Schur convex (concave) if $\quad s=\left(x_{1}-x_{2}\right)\left(\frac{\partial \varphi}{\partial x_{1}}-\frac{\partial \varphi}{\partial x_{2}}\right) \geq 0(\leq 0)$.

(ii) Schur geometrically convex (concave) if $s=\left(\ln x_{1}-\ln x_{2}\right)\left(x_{1} \frac{\partial \varphi}{\partial x_{1}}-x_{2} \frac{\partial \varphi}{\partial x_{2}}\right) \geq 0(\leq 0)$.

(iii) Schur harmonically convex (concave) if $s=(a-b)\left(x_{1}^{2} \frac{\partial \varphi}{\partial x_{1}}-x_{2}^{2} \frac{\partial \varphi}{\partial x_{2}}\right) \geq 0(\leq 0)$.

\section{Schur Properties on Ratio of Difference of Mean}

In this section, the Schur, Schur geometric and Schur harmonically convexities on ratio of difference of mean are established by finding the partial derivatives.

Theorem 3.1. The ratio of difference of means $\frac{M_{C_{d} G H}}{M_{C_{d} A G H}}$ is

(i) Schur convex.

(ii) Schur geometrically convex.

(iii) Schur harmonically convex is for all $a \geq b$.

Proof. Let $\frac{M_{C C_{d} G H}}{M_{C_{d} A G H}}(a, b)=\frac{C(a, b)-C_{d}(a, b)}{C_{d}(a, b)-A(a, b)}$

From lemma 2, Consider, $f(a, b)=C A-C_{d}^{2}=\left(\frac{a^{2}+b^{2}}{a+b}\right)\left(\frac{a+b}{2}\right)-\frac{4}{9}\left(\frac{a^{2}+a b+b^{2}}{a+b}\right)^{2}$ 
By finding the partial derivatives of $f(a, b)$ and with simple manipulation gives

$$
\frac{\partial f}{\partial a}=a-\frac{8}{9}\left(\frac{\left(a^{2}+a b+b^{2}\right) a(a+2 b)}{(a+b)^{3}}\right) \text { and } \frac{\partial f}{\partial b}=b-\frac{8}{9}\left(\frac{\left(a^{2}+a b+b^{2}\right) b(2 a+b)}{(a+b)^{3}}\right)
$$

Proof of (i), from eqn (1), we have

$$
\frac{\partial f}{\partial a}-\frac{\partial f}{\partial b}=(a-b)\left(1-\frac{8\left(a^{2}+a b+b^{2}\right)}{9(a+b)^{2}}\right)=(a-b)\left(\frac{a^{2}+10 a b+b^{2}}{9(a+b)^{2}}\right)
$$

Then $s=(a-b)\left(\frac{\partial f}{\partial a}-\frac{\partial f}{\partial b}\right)=(a-b)^{2}\left(\frac{a^{2}+10 a b+b^{2}}{9(a+b)^{2}}\right) \geq 0$ for all $a \& b$

This verifies the condition for Schur convexity.

Proof of (ii), from eqn (1), we have

$$
a \frac{\partial f}{\partial a}-b \frac{\partial f}{\partial b}=\left(a^{2}-b^{2}\right)-\frac{8}{9}\left(\frac{\left(a^{2}+a b+b^{2}\right)\left(a^{3}-b^{3}+2 a b(a-b)\right)}{(a+b)^{3}}\right) \geq 0 \text { for all } a \& b
$$

Then $s=(\ln a-\ln b)\left(a \frac{\partial f}{\partial a}-b \frac{\partial f}{\partial b}\right)=(\ln a-\ln b) \frac{a-b}{9(a+b)^{3}}\left(a^{4}+b^{4}+14 a^{2} b^{2}+2 a^{3} b+2 a b^{3}\right) \geq 0$

This verifies the condition for Schur geometrically convexity.

Proof of (iii), As above we have

Then $s=(a-b)\left(a^{2} \frac{\partial f}{\partial a}-b^{2} \frac{\partial f}{\partial b}\right)=(a-b) \frac{\left(a^{2}+10 a b+b^{2}\right)}{9(a+b)^{2}} \geq 0$ for $a \& b$

This verifies the condition for Schur Harmonically convex.

Thus the proof of theorem 3.1 is completed.

Theorem 3.2. The ratio of difference of means $\frac{M_{C C_{d} G H}}{M_{A H_{e} G H}}$ is

(i) Schur convex.

(ii) Schur geometrically convex.

(iii) Schur harmonically convex is for all $a \geq b$.

Proof: Let $\frac{M_{C C_{d} G H}}{M_{A H_{e} G H}}(a, b)=\frac{C(a, b)-C_{d}(a, b)}{A(a, b)-H_{e}(a, b)}$

From lemma 2, Consider, $f(a, b)=C_{e}-A C_{d}$

$$
=\left(\frac{a^{2}+b^{2}}{a+b}\right)\left(\frac{a+b+\sqrt{a b}}{3}\right)-\left(\frac{a+b}{2}\right) \frac{2}{3}\left(\frac{a^{2}+a b+b^{2}}{a+b}\right)=\frac{\sqrt{a b}}{3}\left(a+b-\frac{2 a b}{a+b}-\sqrt{a b}\right)
$$


By finding the partial derivatives of $f(a, b)$ and with simple manipulation gives

$$
\begin{aligned}
& \frac{\partial f}{\partial a}=\frac{\sqrt{a b}}{3}\left(1-\frac{2 b^{2}}{(a+b)^{2}}-\frac{\sqrt{b}}{2 \sqrt{a}}\right)+\left(a+b-\frac{2 a b}{a+b}-\sqrt{a b}\right) \frac{\sqrt{b}}{6 \sqrt{a}} \\
& a \frac{\partial f}{\partial a}=\frac{\sqrt{a b}}{6}\left(\left(2 a-\frac{4 a b^{2}}{(a+b)^{2}}-\sqrt{a b}\right)+\left(a+b-\frac{2 a b}{a+b}-\sqrt{a b}\right)\right) \\
& \text { similarly } \quad b \frac{\partial f}{\partial b}=\frac{\sqrt{a b}}{6}\left(\left(2 b-\frac{4 a^{2} b}{(a+b)^{2}}-\sqrt{a b}\right)+\left(a+b-\frac{2 a b}{a+b}-\sqrt{a b}\right)\right)
\end{aligned}
$$

Proof of (i), from eqs (2) \& (3) we have

Then $s=(a-b)\left(\frac{\partial f}{\partial a}-\frac{\partial f}{\partial b}\right)=\frac{(a-b)^{2}}{6(a+b)(\sqrt{a b})}\left(4 a b+2 \sqrt{a b}(a+b)-a^{2}-b^{2}\right) \geq 0$ for $a \& b$.

This verifies the condition for Schur convex.

Proof (ii), from eqs (2) \& (3) we have

$$
a \frac{\partial f}{\partial a}-b \frac{\partial f}{\partial b}=\frac{\sqrt{a b}}{6}\left(2 a-2 b-\frac{4 a b(b-a)}{(a+b)^{2}}\right)
$$

Consider, $s=(\ln a-\ln b)\left(a \frac{\partial f}{\partial a}-b \frac{\partial f}{\partial b}\right)=(\ln a-\ln b) \frac{\sqrt{a b}(a-b)}{3}\left(1+\frac{2 a b}{(a+b)^{2}}\right) \geq 0$ for $a \& b$

This verifies the condition for Schur geometrically convex.

Proof of (iii), from eqs (2) \& (3) we have

$$
a^{2} \frac{\partial f}{\partial a}-b^{2} \frac{\partial f}{\partial b}=\frac{\sqrt{a b}}{6}(a-b)\left(3 a+3 b-2 \sqrt{a b}-\frac{2 a b}{(a+b)}\right)
$$

Then $s=(a-b)\left(a^{2} \frac{\partial f}{\partial a}-b^{2} \frac{\partial f}{\partial b}\right)=\frac{\sqrt{a b}}{6} \frac{(a-b)}{(a+b)}\left(3\left(a^{2}+b^{2}\right)+4 a b-2 \sqrt{a b}(a+b)\right) \geq 0$ for $a \& b$

This verifies the condition for Schur harmonically convex.

Thus the proof of theorem 3.2 is completed.

Theorem 3.3. The ratio of difference of means $\frac{M_{C A G H}}{M_{A H_{e} G H}}$ is

(i) Schur convex.

(ii) Schur geometrically convex.

(iii) Schur harmonically convex is for all $a \geq b$.

Proof: Similar argument as discussed in theorem 3.1 and 3.2 gives the proof of theorem 3.3. 


\section{REFERENCES}

[1] P. S. Bullen, Handbook of means and their inequalities, Kluwer Acad. Publ., Dordrecht, 2003.

[2] G. H. Hardy, J. E. Littlewood and G. Polya, Inequalities, 2nd edition, Cambridge University Press, Cambridge, 1959.

[3] A. M. Marshall and I.Olkin, Inequalities: Theory of Majorization and Its Application, New York : Academies Press, 1979.

[4] K. M. Nagaraja, V. Lokesha and S. Padmanabhan, A simple proof on strengthening and extension of inequalities Adv. Stud. Contemp. Math.,, 17(1)(2008), 97-103.

[5] I. J. Taneja, On a difference of Jensen inequality and its applications to mean divergence measures, RGMIA Research Report Collection, http://rgmia.vu.edu.au,7(4)(2004), Art. 16. in:arXiv:math.PR/0501302

[6] I. J. Taneja, Refinement of inequalities among means, Journal of Combinatorics, Information and System Sciences, 31(1-4)(2006), 343-364.

[7] B. Y. Wang, Foundations of majorization inequalities, Beijing Normal Univ. Press, Beijing, China, 1990(in Chinese).

[8] S. Wang and Y. Chu, The best bounds of combination of arithmetic and harmonic means for the Seiffert's mean, Int. J. Math. Analysis, 22(4) (2010), 1079-1084.

[9] J. Rooin and M. Hassni, Some new inequalities between important means and applications to Ky-Fan types inequalities, Math. Ineq. and Appl., 10(3)(2007),78-81

[10] Naveenkumar. B, Sandeepkumar, V. Lokesha, and K. M. Nagaraja, Ratio of difference of means, International e Journal Mathematics and Engineering, 2(2),(2011)932-936.

[11] Y. M. Chu and X. M. Zhang, The Schur geometrical convexity of the extended mean values, J. Convex Anal., 15(4)2008, 869-890.

[12] N. Elezovic and J. Pecaric, Note on Schur-convex functions, Rocky Mountain Math., 29(1998), 853-856.

[13] Huan Nan Shi, Jain Zang and Da-mao Li, Schur-geometric convexity for difference of means, Applied Mathematics E- Notes, 10(2010), 274-284.

[14] V. Lokesha, K. M. Nagaraja, Naveen Kumar. B and Y-.D.Wu, Schur convexity of Gnan mean for two variables, Notes on Number theory and discrete mathematics, 17(4)(2011), 37-41.

[15] K. M. Nagaraja and P. S. K. Reddy, Logarithmic convexity of double sequence, Secientia Magna, 7(2)(2011), 78-81

[16] J. Sandor, The Schur-convexity of Stolarsky and Gini means, Banach J. Math. Anal., 1(2)(2007), 212-215.

[17] H. N. Shi, Y. M. Jiang and W. D. Jiang, Schur-geometrically concavity of Gini mean, Comp. Math. Appl., 57(2009), 266-274.

[18] H. N. Shi, Schur-convex functions relate to Hadamard-type inequalities, J. Math. Inequal., 1(1)(2007), 127-136.

[19] H. N. Shi, S. H. Wu and F. Qi, An alternative note on the Schur-convexity of the extended mean values, Math. Inequal. Appl., 9(2)(2006), 219-224.

[20] H. N. Shi, M. Bencze, S. H. Wu and D. M. Li, Schur convexity of generalized Heronian means involving two parameters, J. Inequal. Appl., V 2008, Art. ID. 879273, 9 pages doi:10.1155/2008/879273.

[21] X. M. Zang, The Schur geometrical convexity of integral arithmetic mean, Inte. J. Pure Appl. Math., 41(7)(2007), 919- 925. 